\title{
Generating Two Continuous Entangled Microwave Beams Using a dc-Biased Josephson Junction
}

\author{
A. Peugeot, ${ }^{1, *}$ G. Ménard $\odot,{ }^{1}$ S. Dambach,${ }^{2}$ M. Westig, ${ }_{5}^{1}$ B. Kubala, ${ }^{2,3}$ Y. Mukharsky, ${ }^{1}$ C. Altimiras $\odot,{ }^{1}$ P. Joyez,,${ }^{1}$ D. Vion $\odot,{ }^{1}$ \\ P. Roche, ${ }_{1}^{1}$ D. Esteve, ${ }^{1}$ P. Milman, ${ }^{4}$ J. Leppäkangas, ${ }^{5}$ G. Johansson $\odot,{ }^{6}$ M. Hofheinz, ${ }^{7}$ J. Ankerhold, ${ }^{2}$ and F. Portier $\oplus^{1}$ \\ ${ }^{1}$ Université Paris-Saclay, CEA, CNRS, SPEC, 91191 Gif-sur-Yvette, France \\ ${ }^{2}$ Institute for Complex Quantum Systems and IQST, University of Ulm, 89069 Ulm, Germany \\ ${ }^{3}$ Institute of Quantum Technologies, German Aerospace Center (DLR), 89069 Ulm, Germany \\ ${ }^{4}$ Université de Paris, Laboratoire Matériaux et Phénomènes Quantiques, CNRS, 75013 Paris, France \\ ${ }^{5}$ Institute of Physics, Karlsruhe Institute of Technology, 76131 Karlsruhe, Germany \\ ${ }^{6}$ Department of Microtechnology and Nanoscience (MC2), \\ Chalmers University of Technology, SE-412 96 Gothenburg, Sweden \\ ${ }^{7}$ Institut quantique and Département GEGI, Université de Sherbrooke, \\ Sherbrooke JIK 2RI Québec, Canada
}

(Received 10 August 2020; revised 22 April 2021; accepted 11 May 2021; published 13 July 2021)

\begin{abstract}
We show experimentally that a dc-biased Josephson junction in series with two microwave resonators emits entangled beams of microwaves leaking out of the resonators. In the absence of a stationary phase reference for characterizing the entanglement of the outgoing beams, we measure second-order coherence functions to prove the entanglement. The experimental results are found in quantitative agreement with theory, proving that the low-frequency noise of the dc bias is the main limitation for the coherence time of the entangled beams. This agreement allows us to evaluate the entropy of entanglement of the resonators, estimate the entanglement flux at their output, and to identify the improvements that could bring this device closer to a useful bright source of entangled microwaves for quantum-technological applications.
\end{abstract}

DOI: $10.1103 /$ PhysRevX.11.031008

\section{INTRODUCTION}

Although the link between electrical transport and emission of radiation has been understood since the invention of electrical lamps, its complete description in the context of quantum conductors requires a comprehensive treatment of the conductor itself, of the charge reservoirs connected to it, and of the electromagnetic modes of the environment that sustain radiation. Despite numerous achievements [1-7], a full understanding is still missing in the general case. A voltage-biased Josephson junction connected to a small number of modes originally in the vacuum state provides a simple model system for this physics. For a dc bias $V$ smaller than the gap voltage $2 \Delta / e$, no quasiparticle excitation can absorb the energy $2 \mathrm{eV}$ provided by the biasing circuit upon the tunneling of a Cooper pair. As a result, a dc current flows through the junction only if this energy can be absorbed by creating photons in the environmental modes [8-11]. Consequently,

*ambroise.peugeot@gmail.com

Published by the American Physical Society under the terms of the Creative Commons Attribution 4.0 International license. Further distribution of this work must maintain attribution to the author(s) and the published article's title, journal citation, and DOI.
Subject Areas: Mesoscopics, Quantum Physics, Superconductivity the properties of the emitted light depend both on the control voltage and on the coupling of the junction to the modes, described by their impedance $\operatorname{Re}[Z(\nu)]$. Previous experiments have shown that shaping $\operatorname{Re}[Z(\nu)]$ by microwave engineering allows for the creation of various nonclassical states of light [12-14] and have thereby led to the emergence of the field of Josephson photonics. In the case where the junction is coupled to two modes at frequencies $\nu_{a} \neq \nu_{b}$ (see Fig. 1), setting the voltage bias such that $2 \mathrm{eV}=h \nu_{a}+h \nu_{b}$ results in the emission of photon pairs, with one photon created in each mode for each Cooper pair tunneling. The experimental observation of this pair-emission mechanism demonstrated that the beams leaking out of the two resonators have nonclassical population correlations [13] but did not provide information on their quantum phase correlations. Are the two microwave beams entangled? If so, how do we describe precisely and quantify this entanglement? In order to characterize the precise nature of the quantum correlations present in this unique nonclassical two-beam source, we have built a new measurement setup able to probe entanglement between the output microwave beams.

The two resonator fields are coherently driven by the Josephson junction. This driving is described by an effective two-mode squeezing Hamiltonian, resulting in nonlocal quadrature correlations of the emitted light which 


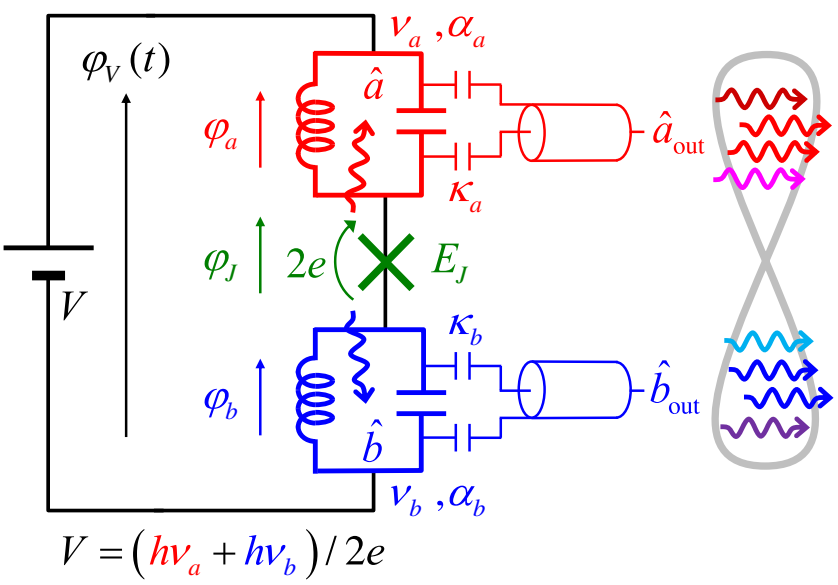

FIG. 1. Principle of the experiment. A simple superconducting circuit made of a Josephson junction (green cross, Josephson energy $E_{J}$ ) in series with two microwave resonators (red and blue $L C$ circuits) having different frequencies $\nu_{a, b}$, similar coupling $\alpha_{a, b}$ to the junction (see text), and similar energy leak rates $\kappa_{a, b}$, is dc biased by a voltage source $V$. Phases $\varphi$ entering the circuit Hamiltonian are also indicated. At a particular voltage (indicated at the bottom), each Cooper pair $2 e$ passing through the junction (green arrow) creates in a coherent way a pair of photons (vertical red and blue wiggles) in the two resonators; entangled microwave fields $a$ and $b$ grow in the resonators, as well as two stationary entangled microwave beams $a_{\text {out }}$ and $b_{\text {out }}$. By measuring correlations between $a_{\text {out }}(t)$ and $b_{\text {out }}(t)$, we experimentally demonstrate this effect.

we evidence experimentally. However, in our experiment, the absence of a well-defined microwave phase reference, due to the dc bias noise and leading to thermal diffusion of the squeezing angle [6], forbids the use of standard techniques to reveal these correlations [15-22]. To demonstrate entanglement in such a scenario without phase stability, i.e., first-order coherence, we rely on the measurement of second-order correlation functions following a detection scheme inspired by Franson interferometry [23]. A simple entanglement witness based on these correlators allows us to prove the entanglement between the outgoing fields.

\section{CIRCUIT MODEL AND PHOTON CORRELATIONS}

The model circuit implemented in this paper is presented in Fig. 1: the series combination of a Josephson junction with Josephson energy $E_{J}$, and of two resonators with different frequencies $\nu_{a, b}$ but similar characteristic impedances $Z_{a, b}$ and energy leak rates $\kappa_{a, b}=2 \pi \nu_{a, b} / Q_{a, b}$, is biased by an ideal dc voltage source $V$. The voltages $v_{x}$ and phases $\varphi_{x}=2 e / \hbar \int v_{x} d t$ across each passive dipole $x$ obey Kirchhoff's law,

$$
\varphi_{J}+\varphi_{a}+\varphi_{b}=\varphi_{V}=2 \pi \nu_{J} t
$$

with $\nu_{J}=2 \mathrm{eV} / \mathrm{h}$ the Josephson frequency.
Up to the zero-point energy of the resonators, the resulting time-dependent Hamiltonian of the circuit reads

$$
\hat{H}=h \nu_{a} \hat{n}_{a}+h \nu_{b} \hat{n}_{b}-E_{J} \cos \left(\hat{\varphi}_{a}+\hat{\varphi}_{b}-2 \pi \nu_{J} t\right)
$$

Here $\hat{n}_{\xi}=\hat{\xi}^{\dagger} \hat{\xi}$ and $\hat{\xi}$ are the photon number and annihilation operators of mode $\xi \in\{a, b\}$, and $\hat{\varphi}_{\xi}=\sqrt{\alpha_{\xi}}\left(\hat{\xi}+\hat{\xi}^{\dagger}\right)$ with $\alpha_{\xi}=\pi Z_{\xi}(2 e)^{2} / h$. The coupling constant $\alpha_{\xi}$ for the interaction between the junction and mode $\xi$ plays the same role as the fine-structure constant in quantum electrodynamics. The $\cos (\varphi)$ term of Eq. (2) may be expanded exactly, yielding an infinite series of terms oscillating at $\nu_{J}+m \nu_{a}+n \nu_{b}$ with $\{m, n\} \in \mathbb{Z}^{2}$. The particular case of interest in this work is the resonance condition $2 e V \simeq h \nu_{a}+h \nu_{b}$, for which the energy delivered by the voltage source for each tunneling Cooper pair is entirely converted into a pair of photons, one in each mode. Moving to a frame rotating at frequency $\nu_{a}+\nu_{b}=\nu_{J}-\delta$ and performing a rotating-wave approximation, for a detuning $\delta$ small enough compared to $\nu_{a}, \nu_{b}$, we arrive at the effective Hamiltonian,

$$
\begin{aligned}
\hat{H}_{\mathrm{RWA}}= & \frac{B}{2}: \sum_{k, l}^{\infty} \frac{(-1)^{k+l}\left(\alpha_{a} \hat{n}_{a}\right)^{k}\left(\alpha_{b} \hat{n}_{b}\right)^{l}}{k ! l !(k+1) !(l+1) !} \\
& \times e^{-2 i \pi \delta t} \hat{a}^{\dagger} \hat{b}^{\dagger}:+ \text { H.c. },
\end{aligned}
$$

in terms of the creation and annihilation operators, or equivalently at

$$
\hat{H}_{\mathrm{RWA}}=\frac{B}{2}\left(e^{-2 i \pi \delta t} \hat{a}^{\dagger} \hat{b}^{\dagger} \hat{L}_{a} \hat{L}_{b}+\text { H.c. }\right) .
$$

Here, $B=E_{J}^{*} \sqrt{\alpha_{a} \alpha_{b}}$, with $E_{J}^{*}=E_{J} e^{-\left(\alpha_{a}+\alpha_{b}\right) / 2}$ is a reduced Josephson energy [14,24-29], the colons indicate normal ordering, and $\hat{L}_{\xi}=\sum_{k=0}^{\infty} L_{k}^{(1)}\left(\alpha_{\xi}\right) /(k+1)|k\rangle\langle k|$ is a diagonal operator in the Fock basis $\{|k\rangle\}$ involving the generalized Laguerre polynomials $L_{k}^{(1)}$. In the regime $\alpha_{\xi} \ll 1$ of weak coupling to the modes, and of low resonator populations $\alpha_{\xi}\left\langle\hat{n}_{\xi}\right\rangle \ll 1$, the $(k, l) \neq(0,0)$ terms may be neglected in Eq. (3) and $\hat{L}_{\xi} \simeq \hat{\mathbb{1}}$ in Eq. (4), so that $\hat{H}_{\mathrm{RWA}}$ reduces to the two-mode squeezing Hamiltonian:

$$
\hat{H}_{\mathrm{TMS}}=\frac{B}{2} e^{-2 i \pi \delta t} \hat{a}^{\dagger} \hat{b}^{\dagger}+\text { H.c. }
$$

The coherent driving term $\hat{a}^{\dagger} \hat{b}^{\dagger}$ with absolute amplitude $B$ creates photon pairs in $a$ and $b$ and entangles the two resonators. The corresponding fields leak out at rates $\kappa_{a, b}$, which brings the resonators in a stationary state and form two entangled beams of light centered on frequencies $\simeq \nu_{a}+\delta / 2$ and $\simeq \nu_{b}+\delta / 2$. A natural dimensionless driving strength is thus $\beta=B / \hbar \sqrt{\kappa_{a} \kappa_{b}}$, proportional to $E_{J}$ and not to the amplitude of an ac drive as in usual two-mode 
squeezers. An input-output model [6] allows us to relate the properties of the outgoing fields $\hat{a}_{\text {out }}(\nu), \hat{b}_{\text {out }}\left(\nu^{\prime}\right)$ to $\beta, \kappa_{a, b}$, and $\delta$. The field quadratures at frequency $\nu$ in the $a$ band are found to be correlated with field quadratures at frequency $\nu^{\prime}=\nu_{J}-\nu$ in the $b$ band: a sum of properly chosen quadratures of $\hat{a}_{\text {out }}(\nu)$ and $\hat{b}_{\text {out }}\left(\nu^{\prime}\right)$ displays fluctuations below their value in the vacuum state. This two-mode squeezing is predicted to be the largest at the maximum of the emission spectral density.

Detection of such a squeezing is usually achieved by filtering the $a_{\text {out }}(\nu)$ and $b_{\text {out }}\left(\nu^{\prime}\right)$ fields in narrow frequency bands and correlating their quadratures after demodulation [15-22]. In the ideal noiseless bias case, a nonzero expectation value of the first-order correlation function (second order in the fields) $\left\langle\hat{a}_{\text {out }} \hat{b}_{\text {out }}(t)\right\rangle \propto e^{-2 i \pi \delta t}$ [where $\hat{\xi}_{\text {out }}(t)=$ $\left.\int \hat{\xi}_{\text {out }}(\nu) e^{2 i \pi \nu t} d \nu\right]$ reveals the correlations between $\hat{a}_{\text {out }}(\nu)$ and $\hat{b}_{\text {out }}\left(\nu^{\prime}\right)$. However, in our experiment we cannot implement this protocol because of the thermal fluctuations of the voltage bias, and consequently of $\delta$, which blur correlations between $\nu$ and $\nu^{\prime}$ faster than we can average them. Instead, we resort to a measurement scheme analog to the one originally developed by Franson [23]. We use the particular second-order correlation function (fourth order in the fields) $\left\langle\hat{a}_{\text {out }}^{\dagger}(t) \hat{b}_{\text {out }}^{\dagger}(t) \hat{a}_{\text {out }}(t+\tau) \hat{b}_{\text {out }}(t+\tau)\right\rangle$ in which the unknown phase of $\hat{a}_{\text {out }}(t+\tau) \hat{b}_{\text {out }}(t+\tau)$ is compensated by the counterrotating phase of $\hat{a}_{\text {out }}^{\dagger}(t) \hat{b}_{\text {out }}^{\dagger}(t)$. This compensation is exact for time delays $\tau$ shorter than the Josephson frequency jitter autocorrelation time. The correlator averages to a finite value for such time delays and makes it possible to define an entanglement witness for the propagating fields. This concept for the detection of entanglement is not limited to the present system and can generally be applied to any system lacking phase stability.

Starting from the theorem demonstrated in Ref. [30], we developed an entanglement witness on the basis of the Franson-type correlator: for any separable state of $a_{\text {out }}$ and $b_{\text {out }}$ the following inequality holds,

$$
\left|g_{\phi}^{(2)}(\tau)\right| \leq g_{a b, \mathrm{sym}}^{(2)}(\tau) \equiv \sqrt{g_{a b}^{(2)}(\tau) \times g_{a b}^{(2)}(-\tau)},
$$

where

$$
g_{\phi}^{(2)}(\tau)=\left\langle\hat{a}_{\text {out }}^{\dagger}(0) \hat{b}_{\text {out }}^{\dagger}(0) \hat{a}_{\text {out }}(\tau) \hat{b}_{\text {out }}(\tau)\right\rangle / D
$$

and

$$
g_{a b}^{(2)}(\tau)=\left\langle\hat{a}_{\text {out }}^{\dagger}(0) \hat{a}_{\text {out }}(0) \hat{b}_{\text {out }}^{\dagger}(\tau) \hat{b}_{\text {out }}(\tau)\right\rangle / D,
$$

with $D=\kappa_{a} n_{a} \kappa_{b} n_{b}$ and $n_{\xi}=\left\langle\hat{n}_{\xi}\right\rangle$. Measuring a violation of inequality (6) is thus a sufficient condition for $a_{\text {out }}$ and $b_{\text {out }}$ to be entangled. Both $\left|g_{\phi}^{(2)}(\tau)\right|$ and $g_{a b \text {,sym }}^{(2)}(\tau)$ initially decay quickly on a scale set by the photon lifetime, which determines for how long photons created by the same tunneling event are observed. After this initial decay $g_{a b \text {,sym }}^{(2)}(\tau) \rightarrow 1$ becomes constant, while the further decay of $\left|g_{\phi}^{(2)}(\tau)\right|$ reflects how phase correlations between $a$ and $b$ modes are scrambled by the low-frequency voltage noise. This implies that the witness can prove entanglement of the outgoing modes only if photons leave the resonator faster than phase coherence is washed out. As previous experiments [14] have shown that the dephasing time expected from the voltage noise across the junction is in the microseconds range in our experimental setup [31], we design our resonator with energy decay times of a few nanoseconds.

Note that the $g_{a b}^{(2)}(\tau)$ correlation function in Eq. (8) is equal to the coherence function that was measured in Ref. [13] in order to prove the nonclassical character of the two emitted beams. In this previous experiment, the correlations between the populations of the two modes were found to be larger than possible in a classical wave theory of light, indicating that light was indeed created as pairs of quanta (photons). This result obtained from power fluctuation measurements was, however, not enough to prove entanglement, which requires measuring the phase coherence of the two beams as in the present work.

\section{EXPERIMENTAL SETUP}

The implementation of our experiment is shown in Fig. 2. The Josephson element is implemented as an $\mathrm{Al} / \mathrm{AlOx} / \mathrm{Al}$ superconducting quantum interferometer device [SQUID; see Fig. 2(b)] whose Josephson energy can be tuned by a magnetic flux applied to its loop. Each resonator at $\sim 5$ and $\sim 7 \mathrm{GHz}$ is made of three cascaded niobium coplanar waveguide segments with different wave impedances [see Fig. 2(a)], which allows reaching high enough impedance of the modes. The sample is anchored at the $15 \mathrm{mK}$ cold stage of a dilution refrigerator and connected [see Fig. 2(c)] to a low-temperature circuitry similar to that described in Ref. [14]. A small currentbiased coil applies the tuning magnetic flux. The sample is voltage biased through two commercial bias tees (black rectangles) connected to a voltage divider fed by a roomtemperature voltage source and heavily filtered at $0.8 \mathrm{~K}$ and $15 \mathrm{mK}$. The two emitted beams are available at the capacitive outputs A and B of the bias tees. They are then routed through filters and isolators to a $-3 \mathrm{~dB}$ hybrid coupler that sends both of them to two nominally identical amplification chains 1 and 2, each equipped with a high electron mobility transistor (HEMT) amplifier. This Hanbury-Brown-Twiss-like setup allows us to reject the uncorrelated noise from the two chains 1 and 2, without affecting the entanglement. Because the $a_{\text {out }}$ and $b_{\text {out }}$ field components along 1 and 2 have well-separated frequencies, they do not interfere and can be processed by the same wideband HEMT. 
(d)
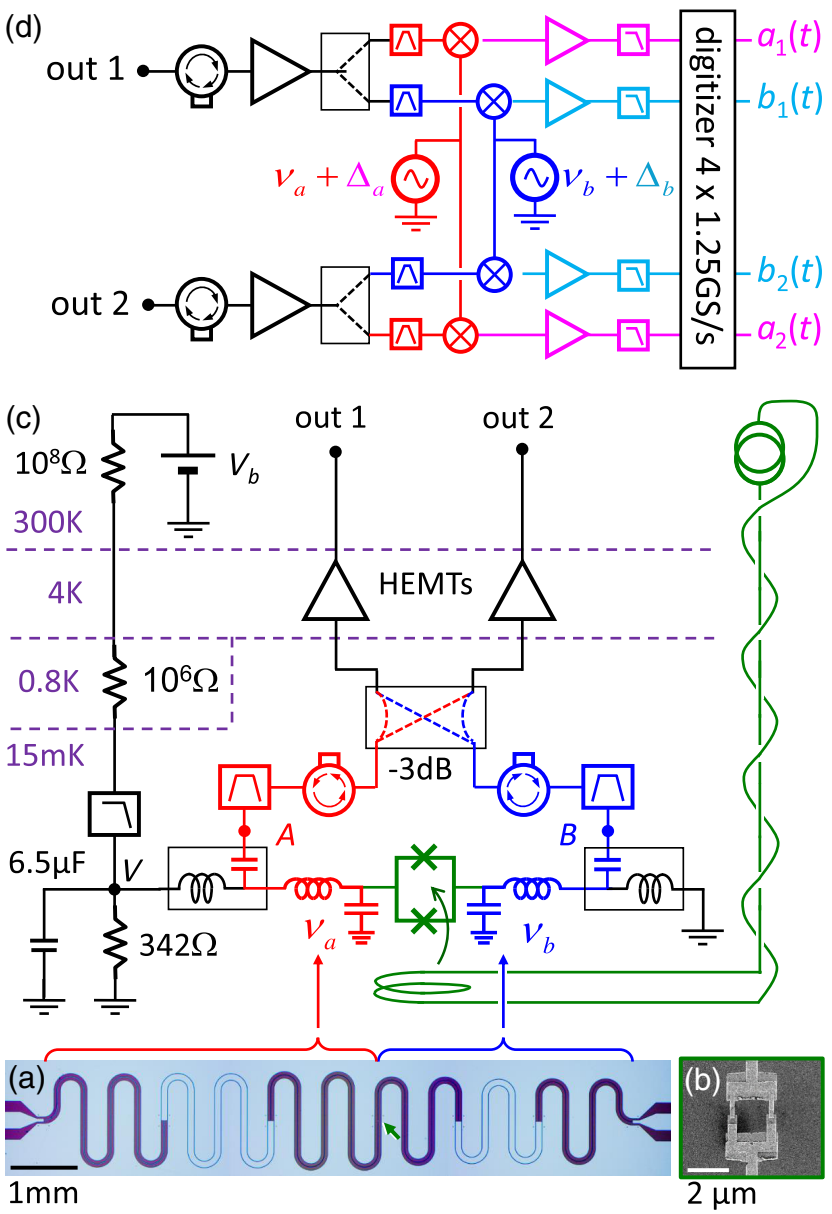

FIG. 2. Implementation of the experiment sketched in Fig. 1. (a) Optical micrograph of the circuit on its silicon chip (see text). The green arrow indicates the position of the SQUID. (b) Scanning electron micrograph of the SQUID used as a tunable Josephson element. (c) Schematic electrical circuit connecting the chip at $15 \mathrm{mK}$ (green SQUID and red and blue $L C$ resonators) to room temperature (see text). (d) Schematic room-temperature setup for heterodyning and digitizing the outgoing fields. The red and blue filters are $\sim 700 \mathrm{MHz}$ wide and centered around $\nu_{a}$ and $\nu_{b}$, respectively. The microwave signals are then down-converted, amplified, and low-pass filtered with a $\sim 600 \mathrm{MHz}$ cutoff frequency and then digitized, as shown by the magenta and cyan symbols.

The room-temperature setup [see Fig. 2(c)] is designed to measure cross-correlation functions of the signals emitted in two frequency bands around $\nu_{a}$ and $\nu_{b}$. Each of the signal outputs 1 and 2 is further amplified and split into the two original microwave components (red and blue), which are then filtered and heterodyned to bring them in the dc $600 \mathrm{MHz}$ frequency range (magenta and cyan). The four temporal signals $\left(a_{1}, b_{1}, a_{2}, b_{2}\right)$ are finally digitized and postprocessed in order to compute power spectral densities (PSDs) and time-domain correlators. The effective acquisition bandwidth of $525 \mathrm{MHz}$ imposed by the whole acquisition chain is chosen to be wide enough to capture most of the short-time features of the correlators in inequality (6), while limiting the noise window and measurement times. A loss of measured entanglement is thus to be expected from this filtering.

\section{RESULTS}

The two resonances at $\nu_{a}=5.092 \mathrm{GHz}$ and $\nu_{b}=$ $6.955 \mathrm{GHz}$ as well as their quality factors $Q_{a}=60.8$ and $Q_{b}=97.0$ are first obtained in situ from the shot noise at bias voltage above the superconducting gap [14]. They lead to similar energy leak rates $\kappa_{a}=5.26 \times 10^{8} \mathrm{~s}^{-1}$ and $\kappa_{b}=4.51 \times 10^{8} \mathrm{~s}^{-1}$. The resonator characteristic impedances yield similar coupling factors $\alpha_{a}=0.070$ and $\alpha_{b}=0.061$.

The transfer functions of the four acquisition chains $\left(a_{1}, b_{1}, a_{2}, b_{2}\right)$ between points $\mathrm{A}$ or $\mathrm{B}$ and the digitizer in Figs. 2(c) and 2(d) are then calibrated. These functions shown in Figs. SM2(a) and SM2(b) of the Supplemental Material (SM) [32] result from the filtering, attenuation, gains, and partial reflections by the different components all along the lines. They have globally the shape of a bandpass filter with $\sim 3 \mathrm{~dB}$ ripples that will slightly distort the measured time-domain correlators. We also measure the real part of the impedance $\operatorname{Re}[Z(\nu)]$ seen by the Josephson element [see Figs. 3(a) and 3(b)], which displays two Lorentzian lines centered at $\nu_{a}$ and $\nu_{b}$, slightly distorted by the spurious reflections mentioned above.

We then measure the radiation emitted by each resonator in single photon processes corresponding to the acJosephson regime, that is when $2 \mathrm{eV} \simeq h \nu_{a}$ or $2 \mathrm{eV} \simeq h \nu_{b}$, with a low enough Josephson energy to ensure that $\alpha_{\xi} n_{\xi} \ll 1$, thus avoiding stimulated emission effects [13]. An example of the corresponding PSDs is shown in Figs. 3(a) and 3(b). Both emission lines display a Gaussian shape with a standard deviation of $\sigma=3.3 \mathrm{MHz}$ corresponding to a $h \sigma / 2 e=6.8 \mathrm{nV} \mathrm{rms}$ bias voltage noise. This $\sigma$ value was observed to decrease slowly in time as the experiment was thermalizing, with sudden rises at each liquid helium transfer in the cryostat. Complementary measurements allowed us to attribute this noise mostly to a parasitic $L C$ mode of the bias tees around $70 \mathrm{kHz}$, expected to yield $\sigma=\sqrt{2 k_{B} T / C_{\text {bias tee }}}$. The lowest observed value $\sigma=2.6 \mathrm{MHz}$ corresponds to a $20 \mathrm{mK}$ temperature of the bias circuit, in reasonable agreement with the $15 \mathrm{mK}$ temperature of the fridge.

We then set the voltage to the targeted resonance condition $2 \mathrm{eV} \simeq h \nu_{a}+h \nu_{b}$, and observe the simultaneous emission around $\nu_{a}$ and $\nu_{b}$, for several driving strengths $\beta$ between 0.4 and 1. Figure 3(c) shows two examples of PSD at $\beta=0.631$ and $\beta=0.905$. The identical shape of the two peaks of the same pair, their common total power $\kappa_{a} n_{a}=$ $\kappa_{b} n_{b}=180$ and $450 \mathrm{Mphotons} / \mathrm{s}$ (yielding an average resonator population $n=\sqrt{n_{a} n_{b}}=0.4$ and 1.5), as well as their larger width compared to the single photon case $2 \mathrm{eV} \simeq h \nu_{a, b}$, reflect the emission by photon-pair 

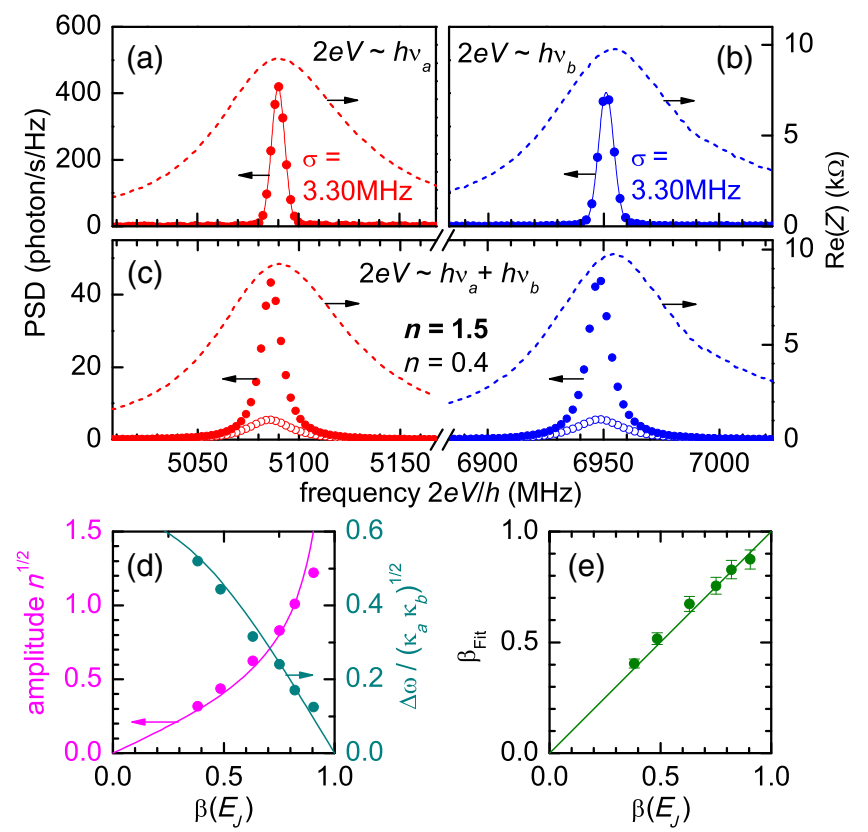

FIG. 3. Characterization of the emission. (a),(b) In situ measured real part of the impedance across the Josephson element (dashed lines, right-hand axis), and example of measured (dots) and Gaussian-fitted (lines) power spectral densities (left-hand axis) recorded separately around $2 \mathrm{eV}=h \nu_{a}$ (a) and $2 \mathrm{eV}=h \nu_{b}$ (b) at small $E_{J}$ yielding $n_{a}=0.34$ and $n_{b}=0.39$, respectively. The emission linewidth results from the voltage-noise-induced distribution $p\left(\nu_{J}=2 \mathrm{eV} / \mathrm{h}\right)$ of the Josephson frequency. The corresponding standard deviation $\sigma$ is the same for both lines but drifts slowly back and forth over hours and days. (c) Same real part of the impedance (dashed lines, right-hand axis) as in (a), and examples of emitted power spectral densities (PSDs, left-hand scales) measured at $2 \mathrm{eV}=h \nu_{a}+h \nu_{b}$ and driving strengths $\beta=$ 0.67 (open circles)and $\beta=0.9$ (solid dots) corresponding to average photon numbers $n=0.4$ and $n=1.5$. (d) Measured (symbols) reduced intraresonator amplitude (magenta, left-hand scale) and emission width (dark cyan, right-hand scale) of the PSDs of Fig. SM2 [32], compared to the corresponding analytical expressions (lines) known for Hamiltonian (5). (e) Dimensionless pumping strength fitted from the same PSD versus the calibrated one.

production. In Sec. II of the SM we show that all the measured PSDs are well reproduced by the analytical expression computed from the pure two-mode squeezing Hamiltonian (5) [32]. As shown by Fig. 3(d), we observe a narrowing of the width of the emission spectra with increasing $\beta$, as commonly observed in ac pumped Josephson parametric amplifiers [19,21,33]. However, contrarily to what occurs in these devices, the peak emission frequencies do not shift with increasing pumping strength [see Fig. 3(c)], provided that the dc voltage $V$ across the junction is kept constant (taking into account the voltage drop across the $342 \Omega$ output resistance of the biasing circuit shown in Fig. 2). This is due to the absence of Kerr and cross-Kerr nonlinearities in the normal-ordered
Hamiltonian (3) [32]. As shown by Fig. 3(e), fitting the frequency width of the emitted radiation with the two-mode squeezing expressions yields a pumping strength $\beta$ in quantitative agreement with the one deduced from a determination of $E_{J}^{*}$ using the ac Josephson effect [32].

The second-order correlators $g_{\phi}^{(2)}(\tau)$ and $g_{a b}^{(2)}(\tau)$ are deduced from the measurements of several correlation functions, as explained in the SM [32]. Figure 4(a) shows the corresponding functions $\left|g_{\phi}^{(2)}\right|$ and $g_{a b, \text { sym }}^{(2)}$ for six driving strengths $\beta=0.4,0.51,0.67,0.75,0.82,0.9$ (and independently measured small detunings $\delta=6.7,2.1,-12.1$, $1.8,-11.1$, and $-11.6 \mathrm{MHz}$ ) leading to the average resonator populations $n=0.10,0.2,0.4,0.7,1$, and 1.5 , respectively. These two correlators coincide at $\tau=0$ as expected from their definition. They both present an initial rapid decay over a timescale set by $n$ and the resonator's lifetime. The correlator $g_{a b \text {,sym }}^{(2)}$ converges to 1 while $\left|g_{\phi}^{(2)}\right|$ subsequently follows a slower Gaussian decay down to zero, as expected from the low-frequency voltage noise already mentioned. The experimental entanglement witness $\left|g_{\phi}^{(2)}\right|-g_{a b, \text { sym }}^{(2)}>0$ at short time testifies that the two beams at 5 and $7 \mathrm{GHz}$ are entangled. When increasing $\beta$, entanglement was detected up to $n=5$ (data not shown), although full numerical simulations could not be performed at such high occupation numbers (see Sec. V of SM [32]).

\section{ENTANGLEMENT ANALYSIS}

In order to probe our theoretical modeling of the system dynamics and measurement schemes, we perform numerical quantum simulations of the dynamics of the circuit using the parameters measured in the experiment. The steady-state density matrix $\rho$ of the two resonators is obtained in the Fock state basis using the Lindblad master equation [34] corresponding to Hamiltonian (3) and to the relaxation superoperators $\sqrt{\kappa_{a}} \hat{a}$ and $\sqrt{\kappa_{b}} \hat{b}$. This allows us to check that the field departs negligibly from Gaussian statistics up to 1 photon in each resonator. Then, using the quantum regression theorem $[35,36]$, we simulate the dimensionless correlators $\left\langle\hat{b}^{\dagger}(\tau) \hat{a}^{\dagger}(0) \hat{a}(0) \hat{b}(\tau)\right\rangle /\left(n_{a} n_{b}\right)$ and $\left\langle\hat{a}^{\dagger}(0) \hat{b}^{\dagger}(0) \hat{a}(\tau) \hat{b}(\tau)\right\rangle /\left(n_{a} n_{b}\right)$ (see Sec. V of SM [32]). Using the standard input-output theory [37], one can show that these simulated intraresonator correlators are actually equal to the expressions (7) and (8) for the outgoing fields, immediately at the resonator outputs. We thus plot in dashed lines the simulated $\left|g_{\phi}^{(2)}(\tau)\right|$ functions in the panels of Fig. 4(a), and observe that they are significantly above the measured ones at all times. This is due to the already mentioned frequency filtering by the lines, which has to be included in the simulations. As explained in Sec. V of the SM, this can be done by convoluting simulated four-time $g_{\phi}^{(2)}$ correlators with the four transfer functions of the lines (in the time domain) 

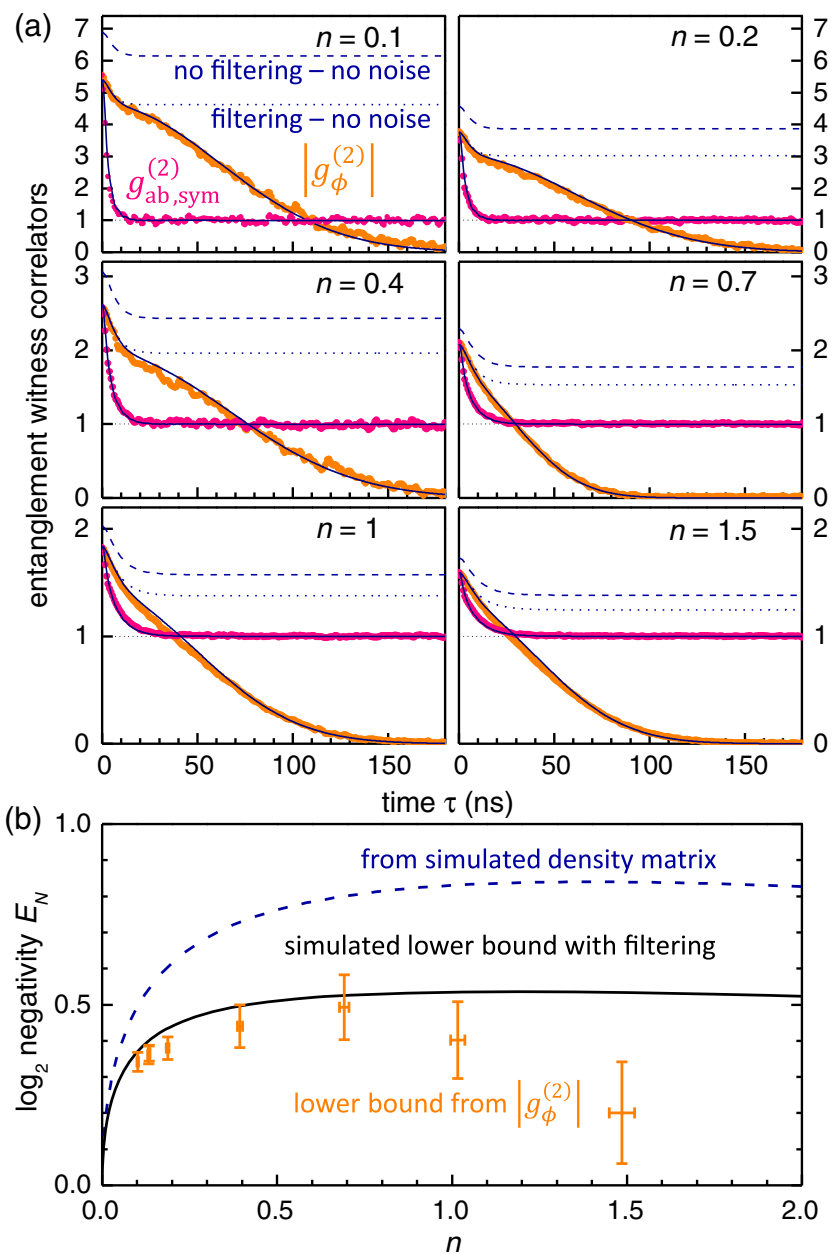

FIG. 4. Demonstration of entanglement. (a) Measured (dots) and simulated (solid lines) two-times fourth order in field correlators $g_{a b, \text { sym }}^{(2)}$ and $g_{\phi}^{(2)}$ (see text) as a function of delay $\tau$, for different Josephson energies leading to the indicated population $n$. Dashed and dotted lines correspond to simulations without voltage noise and with (dotted) and without (dashed) filtering by the measuring lines. (b) $\log _{2}$ negativity of the intraresonator fields as a function of population $n$. Exact value obtained directly from the numerically simulated density matrix of the system (dashed line), and experimental (error bars) and simulated (solid line) lower bound value corresponding to the filtering by the measuring lines (see text).

[32]. But because such a rigorous program would result in much too long simulation times, we use a two-time correlator approach by applying to all lines the same filtering function chosen as the average of the four symmetrized transfer functions [see Figs. SM2(a) and SM2(b) [32]]. We now obtain the dotted lines $\left|g_{\phi \text {,filter }}^{(2)}(\tau)\right|$, which are in good agreement with the experiment at short time, but do not decay to zero at long times. Finally, we also account for the low-frequency voltage fluctuations: we model them as a purely static $\delta$ noise with Gaussian distribution and standard deviation $\sigma$, and compute the weighted average of $g_{\phi \text {,filter }}^{(2)}(\tau)$ over $\delta$. To a very good approximation, this averaging simply multiplies the simulated value by $\exp \left[-2(\pi \sigma \tau)^{2}\right]$. As $\sigma$ was observed to vary slowly in time, we use it as the only fitting parameter to fit each experimental $g_{\phi}^{(2)}(\tau)$. The fits shown in Fig. 4(a) are now satisfactory at all times and yield $\sigma=2.57,2.61,2.40,4.98,3.10$, and $3.64 \mathrm{MHz}$ (in order of increasing $\beta$ or $n$ ). This good agreement validates our model of voltage-noise-induced slow dephasing: on a timescale shorter than $\tau_{\text {bias tee }} \sim \sqrt{L_{\text {bias tee }} C_{\text {bias tee }}}=2.3 \mu$ s, the pump frequency can be considered as fixed and the junction creates a standard two-mode squeezed state in the resonators.

\section{LOG NEGATIVITY AND ENTANGLEMENT WITNESS}

The amount of entanglement between two subsystems $a$ and $b$ can be quantified by their log negativity [38],

$$
E_{N}=\log _{2}\left(\left\|\rho^{T_{b}}\right\|_{1}\right)
$$

where $\rho^{T_{b}}$ is the partial transpose of the density operator $\rho$ of the total system with respect to subsystem $b$, and $\|.\|_{1}$ is the trace norm. It is an upper bound on the number of entangled bits (ebits) that can be distilled from the system. This quantity is directly available from our numerical simulations of the intraresonator fields. It is plotted as a function of $n$ in Fig. 4(b): $E_{N}$ increases rapidly to a very flat maximum of about 0.8 ebits between $n=1$ and 2 photons.

In the case of a two-mode squeezed Gaussian state of $a$ and $b, E_{N}$ can be linked to observable quantities through

$E_{N}=\log _{2}\left[1+n_{a}+n_{b}-\sqrt{\left(n_{a}-n_{b}\right)^{2}+4|\langle a b\rangle|^{2}}\right]$.

We first checked that in our simulations using the full Hamiltonian Eq. (3), the values of $E_{N}$ computed using Eq. (10) agreed with the one computed using Eq. (9) with minute deviations. This allows us to deduce $E_{N}$ from the $g_{\phi}^{(2)}(\tau)$ function measured on the outgoing fields. For a noiseless voltage bias, the correlator in Eq. (10) is given by $\left|\left\langle\hat{a}_{\text {out }} \hat{b}_{\text {out }}\right\rangle\right|^{2}=\kappa_{a} \kappa_{b}|\langle\hat{a} \hat{b}\rangle|^{2}=n_{a} n_{b} g_{\phi}^{(2)}(\tau \rightarrow \infty)$. Although the measured $g_{\phi}^{(2)}(\tau \rightarrow \infty)$ vanishes due to quasistatic noise, we can retrieve its corresponding noiseless values by dividing it by the Gaussian envelopes fitted above (see SM Sec. III [32]). The corresponding apparent log negativities [shown as error bars in Fig. 4(b)] are $40 \%$ below the theoretical estimate (blue dashed line). This discrepancy is again due to the filtering by the lines, which cuts out part of the quantum correlations, and makes our measured apparent $\log$ negativity only a lower bound of $E_{N}$. This is confirmed by simulations of $\left\langle\hat{a}_{\text {out }} \hat{b}_{\text {out }}\right\rangle$ taking into account the measured filtering, which yields the $E_{N}(n)$ curve shown by the black solid line, in better overall agreement with the measurements. However, the two points at 1 and 1.5 photons are markedly below our prediction, 
which we attribute to a stronger coupling of the Josephson junction to its low-frequency environment. Indeed, when the detuning $\delta$ is positive (negative), the dc differential conductance of the junction happens to be negative (positive), which amplifies (cools down) the low-frequency noise of its environment. At positive detuning (and even zero detuning in the presence of large voltage fluctuations), the amplifying effect makes the system unstable and hysteretic, especially at large drive amplitude $\beta$. To avoid this instability, we actually applied slightly negative detunings to measure the points at $n=1$ and $n=1.5$. The junction then cools down the low-frequency environment [39], converting its energy to the much higher frequencies $\nu_{a, b}$, and degrading the detected entanglement. A systematic and quantitative investigation of this effect goes beyond the scope of the present work, and is left for further investigations.

Although a quantitative account could be reached for the entanglement witness probed, the absence of a stable phase reference for the two-mode squeezing does not allow us to measure sufficiently rapidly the entanglement entropy of each pair of outgoing modes $\left(\nu, \nu^{\prime}=\nu_{J}-\nu\right)$ [40]. It is nevertheless interesting to estimate the instantaneous flux of entanglement entropy, during microsecond-long stability periods. We use for this purpose the two-mode squeezing Hamiltonian model (5) that yielded emission [see Figs. 3(d) and 3(e)] and entanglement properties (see Fig. 4) in agreement with the experimental data. As described in the SM [32], we calculate the entanglement spectral density $E_{N}(\nu)$ defined in Ref. [40] or the related density of entropy of formation $E_{F}(\nu)$ [41], and integrate it over the emission bandwidth $\Delta \nu$. This procedure yields total entanglement fluxes $\Gamma_{N}(\nu)$ and $\Gamma_{F}(\nu)$ at the outputs of the resonators reaching up to about 115 and 103 Mebit/s, respectively. However, we stress here that this appealing figure of merit compared to the one achieved with Josephson parametric amplifiers or converters $[19,22,42]$ is only an estimate. Because of the diffusion of the squeezing angle, it is moreover not directly exploitable for encoding quantum information in continuous variables using presently known protocols. A possible way to directly detect the entanglement between the $\left(\nu, \nu^{\prime}\right)$ outgoing modes would be to incorporate an additional Josephson junction sharing the same dc bias and to use its Josephson radiation as a phase reference.

\section{DISCUSSION}

We now discuss the advantages and limitations of our circuit and its potential developments. We first stress that our dc-biased circuit does not suffer from the Kerr nonlinearities governing the saturation of ac-pumped Josephson parametric amplifiers and converters [19, 21,33]. The nonlinearity of Hamiltonian (3) is of a different nature and is always easy to push to higher photon numbers, allowing for a higher entanglement brightness: in our experiment, the Gaussian character of the emitted light was ensured up to only $1-1.5$ photons in the resonators $\left(\alpha_{\xi} n_{\xi} \ll 1\right)$, but lowering by a factor 10 the impedance of the two-modes (and consequently of the couplings $\alpha_{a, b}$ ), while increasing the Josephson energy $E_{J}$ by the same factor, one could make our entangled microwave source 10 times brighter. Conversely, coupling a dcbiased Josephson junction to several high impedance resonators such as the one described in Ref. [14] would generate highly non-Gaussian entangled beams of photons [43], and combining a high and a low impedance mode has been predicted to stabilize a Fock state of the high impedance mode with a mere dc bias using the two photons processes exploited in this paper [44]. Using Josephson junctions based on superconductors with larger energy gaps (like $\mathrm{NbN}$ ) [12] should allow one to observe the entanglement evidenced here up to terahertz frequencies. Then, transforming our circuit into a source of entangled beams useful for continuous variables protocols requires maintaining the stability of the squeezed two-mode quadrature over long enough times. This implies a significant reduction of the noise on the bias voltage $V$, which could be done on chip using a Shapiro voltage step of an additional Josephson junction. Last, we note that our device can be useful for phase-insensitive applications, such as twophoton light sources for quantum illumination [45].

In a broader picture, it is worth noting that the entanglement of the outgoing modes originates from successive two-photon emission processes associated to Cooper pair tunneling between superconducting condensates with a controlled superconducting phase difference. The properties of the radiation emitted by an out-of-equilibrium quantum conductor arise from charge quantization and from the quantum correlations of its electronic reservoirs. The present work is thus a prime example of the emerging field of mesoscopic quantum electrodynamics of coherent conductors where numerous interesting phenomena were already recently predicted and demonstrated for producing e.g., sub-Poissonian photon sources [12,14,46-50], novel types of lasers [51-54], near-quantum-limited amplifiers [55,56], squeezed radiation [2,3,5,57], or new types of qubits [58], or two-photon losses [59].

As a conclusion, by measuring an entanglement witness, we have shown that when combined with a purely classical voltage source, a simple Josephson junction in series with two resonators can emit two continuous entangled microwave beams at different frequencies. We extracted a lower bound on the value of the logarithmic negativity of the two resonator fields, and showed that our experiment implements a simple and bright source of entangled microwave light beams. This new method for the detection of twomode entanglement in the absence of a known phase reference could be adapted to probe other quantum systems lacking first-order coherence. 


\section{ACKNOWLEDGMENTS}

We gratefully acknowledge stimulating discussions with C. Padurariu, S. Wölk, A. Aspect, E. Flurin, F. Grosshans, and N. Treps, and technical help from P. Jacques. This work received funding from the European Research Council under the European Union's Program for Research and Innovation (Horizon 2020)/ERC Grant Agreement No. 639039. We gratefully acknowledge partial support from LabEx PALM (ANR-10-LABX-0039-PALM), ANR contract GEARED (ANR-14-CE26-0018-01) and SIMCIRCUIT (ANR-18-CE47-0014-01), from the ANRDFG Grant JosephCharli, and from the ERC through the NSECPROBE grant, from IQST and the German Science Foundation (DFG) through AN336/11-1. S. D. acknowledges financial support by the Carl Zeiss Foundation and the German Academic Exchange Service (DAAD).

It is with the deepest sorrow and gratitude that we acknowledge especially the inspiration for, and contribution to, this work from its principal investigator and our friend, Fabien Portier. His untimely passing in December, 2020, at the age of 45 is a big loss to the mesoscopic physics community.

[1] A. Cottet, T. Kontos, and B. Douçot, Electron-Photon Coupling in Mesoscopic Quantum Electrodynamics, Phys. Rev. B 91, 205417 (2015).

[2] O. Dmytruk, M. Trif, C. Mora, and P. Simon, Out-ofEquilibrium Quantum Dot Coupled to a Microwave Cavity, Phys. Rev. B 93, 075425 (2016).

[3] C. Mora, C. Altimiras, P. Joyez, and F. Portier, Quantum Properties of the Radiation Emitted by a Conductor in the Coulomb Blockade Regime, Phys. Rev. B 95, 125311 (2017).

[4] C. Altimiras, F. Portier, and P. Joyez, Interacting Electrodynamics of Short Coherent Conductors in Quantum Circuits, Phys. Rev. X 6, 031002 (2016).

[5] A. L. Grimsmo, F. Qassemi, B. Reulet, and A. Blais, Quantum Optics Theory of Electronic Noise in Coherent Conductors, Phys. Rev. Lett. 116, 043602 (2016).

[6] J. Leppäkangas, G. Johansson, M. Marthaler, and M. Fogelström, Input-Output Description of Microwave Radiation in the Dynamical Coulomb Blockade, New J. Phys. 16, 015015 (2014).

[7] J. Leppäkangas, G. Johansson, M. Marthaler, and M. Fogelström, Nonclassical Photon Pair Production in a Voltage-Biased Josephson Junction, Phys. Rev. Lett. 110, 267004 (2013).

[8] D. V. Averin, Yu. V. Nazarov, and A. A. Odintsov, Incoherent tunneling of the cooper pairs and magnetic flux quanta in ultrasmall Josephson junctions, Physica (Amsterdam) 165-166B, 945 (1990).

[9] G.-L. Ingold and Y. V. Nazarov, Charge Tunneling Rates in Ultrasmall Junctions, in Single Charge Tunneling, edited by H. Grabert and M. H. Devoret (Plenum, New York, 1992).
[10] T. Holst, D. Esteve, C. Urbina, and M. H. Devoret, Effect of a Transmission Line Resonator on a Small Capacitance Tunnel Junction, Phys. Rev. Lett. 73, 3455 (1994).

[11] M. Hofheinz, F. Portier, Q. Baudouin, P. Joyez, D. Vion, P. Bertet, P. Roche, and D. Estève, Bright Side of the Coulomb Blockade, Phys. Rev. Lett. 106, 217005 (2011).

[12] A. Grimm, F. Blanchet, R. Albert, J. Leppäkangas, S. Jebari, D. Hazra, F. Gustavo, J.-L. Thomassin, E. Dupont-Ferrier, F. Portier, and M. Hofheinz, Bright OnDemand Source of Antibunched Microwave Photons Based on Inelastic Cooper Pair Tunneling, Phys. Rev. X 9, 021016 (2019).

[13] M. Westig, B. Kubala, O. Parlavecchio, Y. Mukharsky, C. Altimiras, P. Joyez, D. Vion, P. Roche, D. Esteve, M. Hofheinz, M. Trif, P. Simon, J. Ankerhold, and F. Portier, Emission of Nonclassical Radiation by Inelastic Cooper Pair Tunneling, Phys. Rev. Lett. 119, 137001 (2017).

[14] C. Rolland, A. Peugeot, S. Dambach, M. Westig, B. Kubala, Y. Mukharsky, C. Altimiras, H. le Sueur, P. Joyez, D. Vion, P. Roche, D. Esteve, J. Ankerhold, and F. Portier, Antibunched Photons Emitted by a dc-Biased Josephson Junction, Phys. Rev. Lett. 122, 186804 (2019).

[15] T. Debuisschert, S. Reynaud, A. Heidmann, E. Giacobino, and C. Fabre, Observation of Large Quantum Noise Reduction Using an Optical Parametric Oscillator, Quantum Opt. 1, 3 (1989).

[16] A. Heidmann, R. J. Horowicz, S. Reynaud, E. Giacobino, C. Fabre, and G. Camy, Observation of Quantum Noise Reduction on Twin Laser Beams, Phys. Rev. Lett. 59, 2555 (1987).

[17] G. Brida, L. Caspani, A. Gatti, M. Genovese, A. Meda, and I. R. Berchera, Measurement of Sub-Shot-Noise Spatial Correlations without Background Subtraction, Phys. Rev. Lett. 102, 213602 (2009).

[18] J.-C. Forgues, C. Lupien, and B. Reulet, Emission of Microwave Photon Pairs by a Tunnel Junction, Phys. Rev. Lett. 113, 043602 (2014).

[19] E. Flurin, N. Roch, F. Mallet, M. H. Devoret, and B. Huard, Generating Entangled Microwave Radiation Over Two Transmission Lines, Phys. Rev. Lett. 109, 183901 (2012).

[20] E. Flurin, The Josephson Mixer: A Swiss Army Knife for Microwave Quantum Optics, Ph.D. thesis, École Normale Supérieure, 2014.

[21] C. Eichler, Y. Salathe, J. Mlynek, S. Schmidt, and A. Wallraff, Quantum-Limited Amplification and Entanglement in Coupled Nonlinear Resonators, Phys. Rev. Lett. 113, 110502 (2014).

[22] B. H. Schneider, A. Bengtsson, I. M. Svensson, T. Aref, G. Johansson, J. Bylander, and P. Delsing, Observation of Broadband Entanglement in Microwave Radiation from a Single Time-Varying Boundary Condition, Phys. Rev. Lett. 124, 140503 (2020).

[23] J. D. Franson, Bell Inequality for Position and Time, Phys. Rev. Lett. 62, 2205 (1989).

[24] G. Schn and A. D. Zaikin, Quantum Coherent Effects, Phase Transitions, and the Dissipative Dynamics of Ultra Small Tunnel Junctions, Phys. Rep. 198, 237 (1990).

[25] H. Grabert, G.-L. Ingold, and B. Paul, Phase Diffusion and Charging Effects in Josephson Junctions, Europhys. Lett. 44, 360 (1998). 
[26] P. Joyez, Self-Consistent Dynamics of a Josephson Junction in the Presence of an Arbitrary Environment, Phys. Rev. Lett. 110, 217003 (2013).

[27] V. Gramich, B. Kubala, S. Rohrer, and J. Ankerhold, From Coulomb-Blockade to Nonlinear Quantum Dynamics in a Superconducting Circuit with a Resonator, Phys. Rev. Lett. 111, 247002 (2013).

[28] S. Dambach, B. Kubala, V. Gramich, and J. Ankerhold, Time-Resolved Statistics of Nonclassical Light in Josephson Photonics, Phys. Rev. B 92, 054508 (2015).

[29] J. P. Martínez, S. Léger, N. Gheeraert, R. Dassonneville, L. Planat, F. Foroughi, . Krupko, O. Buisson, C. Naud, W. Hasch-Guichard, S. Florens, I. Snyman, and N. Roch, A Tunable Josephson Platform to Explore Many-Body Quantum Optics in Circuit-QED, npj Quantum Inf. 5, 1 (2019).

[30] S. Wölk, M. Huber, and O. Gühne, Unified Approach to Entanglement Criteria Using the Cauchy-Schwarz and Hölder Inequalities, Phys. Rev. A 90, 022315 (2014).

[31] More precisely, previous experiments showed that the linewidth of the one-photon emission process could be as small as $1 \mathrm{MHz}$, yielding a phase coherence time of about $1 \mu \mathrm{s}$. We then expected the two-photon phase coherence time to have a similar value in this experiment.

[32] See Supplemental Material at http://link.aps.org/ supplemental/10.1103/PhysRevX.11.031008 for more details about the fabrication process and measurement procedure.

[33] N. Roch, E. Flurin, F. Nguyen, P. Morfin, P. CampagneIbarcq, M. H. Devoret, and B. Huard, Widely Tunable, Nondegenerate Three-Wave Mixing Microwave Device Operating Near the Quantum Limit, Phys. Rev. Lett. 108, 147701 (2012).

[34] A. D. Armour, B. Kubala, and J. Ankerhold, Josephson Photonics with a Two-Mode Superconducting Circuit, Phys. Rev. B 91, 184508 (2015).

[35] C. W. Gardiner and P. Zoller, Quantum Noise: A Handbook of Markovian and Non-Markovian Quantum Stochastic Methods with Applications to Quantum Optics (Springer, Berlin, 2004).

[36] H.-P. Breuer and F. Petruccione, The Theory of Open Quantum Systems (Oxford University Press, Oxford, 2002).

[37] B. Yurke, Input-Output Theory, in Quantum Squeezing, edited by P. D. Drummond and Z. Ficek (Springer, Berlin, 2004).

[38] R. Horodecki, P. Horodecki, M. Horodecki, and K. Horodecki, Quantum Entanglement, Rev. Mod. Phys. 81, 865 (2009).

[39] P. P. Hofer, M. Perarnau-Llobet, J. B. Brask, R. Silva, M. Huber, and N. Brunner, Autonomous Quantum Refrigerator in a Circuit QED Architecture Based on a Josephson Junction, Phys. Rev. B 94, 235420 (2016).

[40] Z. J. Deng, S. J. M. Habraken, and F. Marquardt, Entanglement Rate for Gaussian Continuous Variable Beams, New J. Phys. 18, 063022 (2016).

[41] G. Adesso and F. Illuminati, Gaussian Measures of Entanglement versus Negativities: Ordering of Two-Mode Gaussian States, Phys. Rev. A 72, 032334 (2005).

[42] K. G. Fedorov, S. Pogorzalek, U. Las Heras, M. Sanz, P. Yard, P. Eder, M. Fischer, J. Goetz, E. Xie, K. Inomata, Y. Nakamura, R. Di Candia, E. Solano, A. Marx, F. Deppe, and
R. Gross, Finite-Time Quantum Entanglement in Propagating Squeezed Microwaves, Sci. Rep. 8, 6416 (2018).

[43] C. W. S. Chang, C. Sabín, P. Forn-Díaz, F. Quijandría, A. M. Vadiraj, I. Nsanzineza, G. Johansson, and C. M. Wilson, Observation of Three-Photon Spontaneous Parametric Down-Conversion in a Superconducting Parametric Cavity, Phys. Rev. X 10, 011011 (2020).

[44] J.-R. Souquet and A. A. Clerk, Fock-State Stabilization and Emission in Superconducting Circuits Using dc-Biased Josephson Junctions, Phys. Rev. A 93, 060301(R) (2016).

[45] S. Lloyd, Enhanced Sensitivity of Photodetection via Quantum Illumination, Science 321, 1463 (2008).

[46] C. W. J. Beenakker and H. Schomerus, Counting Statistics of Photons Produced by Electronic Shot Noise, Phys. Rev. Lett. 86, 700 (2001).

[47] C. W. J. Beenakker and H. Schomerus, Antibunched Photons Emitted by a Quantum Point Contact Out of Equilibrium, Phys. Rev. Lett. 93, 096801 (2004).

[48] I. C. Fulga, F. Hassler, and C. W. J. Beenakker, Nonzero Temperature Effects on Antibunched Photons Emitted by a Quantum Point Contact Out of Equilibrium, Phys. Rev. B 81, 115331 (2010).

[49] F. Hassler and D. Otten, Second-Order Coherence of Microwave Photons Emitted by a Quantum Point Contact, Phys. Rev. B 92, 195417 (2015).

[50] W. T. Morley, A. Di Marco, M. Mantovani, P. Stadler, W. Belzig, G. Rastelli, and A. D. Armour, Theory of Double Cooper-Pair Tunneling and Light Emission Mediated by a Resonator, Phys. Rev. B 100, 054515 (2019).

[51] M. C. Cassidy, A. Bruno, S. Rubbert, M. Irfan, J. Kammhuber, R. N. Schouten, A. R. Akhmerov, and L. P. Kouwenhoven, Demonstration of an ac Josephson Junction Laser, Science 355, 939 (2017).

[52] F. Godschalk, F. Hassler, and Y. V. Nazarov, Proposal for an Optical Laser Producing Light at Half the Josephson Frequency, Phys. Rev. Lett. 107, 073901 (2011).

[53] F. Godschalk and Y. V. Nazarov, Lasing at Half the Josephson Frequency with Exponentially Long Coherence Times, Phys. Rev. B 87, 094511 (2013).

[54] F. Godschalk and Y. V. Nazarov, Light-Superconducting Interference Devices, Phys. Rev. B 89, 104502 (2014).

[55] S. Jebari, F. Blanchet, A. Grimm, D. Hazra, R. Albert, P. Joyez, D. Vion, D. Estve, F. Portier, and M. Hofheinz, NearQuantum-Limited Amplification from Inelastic Cooper-Pair Tunnelling, National electronics review 1, 223 (2018).

[56] U. C. Mendes, S. Jezouin, P. Joyez, B. Reulet, A. Blais, F. Portier, C. Mora, and C. Altimiras, Parametric Amplification and Squeezing with an ac-and dc-Voltage Biased Superconducting Junction, Phys. Rev. Applied 11, 034035 (2019).

[57] J.-C. Forgues, C. Lupien, and B. Reulet, Experimental Violation of Bell-like Inequalities by Electronic Shot Noise, Phys. Rev. Lett. 114, 130403 (2015).

[58] J. Estève, M. Aprili, and J. Gabelli, Quantum Dynamics of a Microwave Resonator Strongly Coupled to a Tunnel Junction, arXiv:1807.02364.

[59] A. Cottet and Z. Leghtas, Theory of Interactions between Cavity Photons Induced by a Mesoscopic Circuit, Phys. Rev. B 102, 155105 (2020). 